\section{OPEN ACCESS}

Edited by:

Teodora Handjieva-Darlenska, Independent Researcher,

Sofia, Bulgaria

Reviewed by:

Mohammed Srour.

University of Palestine, Palestine

Sonia Baig,

National University of

Singapore, Singapore

*Correspondence:

Thomas S. Webberley

tomw@cultech.co.uk

${ }^{\dagger}$ Present address

Giulia Masetti,

Department of Cellular Computational and Integrative Biology, University of

Trento, Povo, Italy

Specialty section: This article was submitted to Nutrition and Metabolism, a section of the journal

Frontiers in Nutrition

Received: 16 September 2021 Accepted: 02 November 2021 Published: 25 November 2021

Citation:

Webberley TS, Masetti G, Baker LM,

Dally J, Hughes TR, Marchesi JR, Jack $A$ A, Plummer $S F$, Ramanathan $G$, Facey PD and Michael DR (2021) The Impact of Lab4 Probiotic

Supplementation in a 90-Day Study in Wistar Rats. Front. Nutr. 8:778289. doi: 10.3389/fnut.2021.778289

\title{
The Impact of Lab4 Probiotic Supplementation in a 90-Day Study in Wistar Rats
}

\author{
Thomas S. Webberley ${ }^{1 *}$, Giulia Masetti ${ }^{1+}$, Laura M. Baker ${ }^{2}$, Jordanna Dally ${ }^{1}$, \\ Timothy R. Hughes ${ }^{3}$, Julian R. Marchesi ${ }^{4}$, Alison A. Jack ${ }^{1}$, Sue F. Plummer ${ }^{1}$, \\ Guru Ramanathan ${ }^{5}$, Paul D. Facey ${ }^{2}$ and Daryn R. Michael ${ }^{1}$
}

${ }^{1}$ Cultech Limited, Port Talbot, United Kingdom, ${ }^{2}$ Swansea University Medical School, Swansea University, Swansea, United Kingdom, ${ }^{3}$ Division of Infection and Immunity, School of Medicine, Cardiff University, Cardiff, United Kingdom, ${ }^{4}$ Division of Digestive Diseases, Department of Metabolism, Digestion and Reproduction, Faculty of Medicine, Imperial College London, London, United Kingdom, ${ }^{5}$ Pharmacology based Clinical Trials, Pennington Biomedical Research Centre, Baton Rouge, LA, United States

The anti-inflammatory and cholesterol lowering capabilities of probiotic bacteria highlight them as potential prophylactics against chronic inflammatory diseases, particularly cardiovascular disease. Previous studies in silico, in vitro, and in vivo suggest that the Lab4 probiotic consortium may harbour such capabilities and in the current study, we assessed plasma levels of cytokines/chemokines, short chain fatty acids and lipids and faecal levels of bile acids in a subpopulation of healthy Wistar rats included in 90-day repeat dose oral toxicity study. In the rats receiving Lab4, circulating levels of pro-inflammatory interleukin-6, tumour necrosis factor- $\alpha$ and keratinocyte chemoattractant/growth regulated oncogene were significantly lower compared to the control group demonstrating a systemic anti-inflammatory effect. These changes occurred alongside significant reductions in plasma low density lipoprotein cholesterol and increases in faecal bile acid excretion implying the ability to lower circulating cholesterol via the deconjugation of intestinal bile acids. Correlative analysis identified significant associations between plasma tumour necrosis factor- $\alpha$ and the plasma total cholesterol:high density lipoprotein cholesterol ratio and faecal levels of bifidobacteria in the Lab4 rats. Together, these data highlight Lab4 supplementation as a holistic approach to CVD prevention and encourages further studies in humans.

Keywords: probiotic, inflammation, SCFA, cholesterol, bile, cardiovascular

\section{INTRODUCTION}

The trillions of microorganisms residing in the human gut (the gut microbiota) are intrinsically linked to the well-being of the host (1). Perturbations in the microbiota have been linked with a range of diseases although it is often unclear if this is a causative factor or an epiphenomenon and stabilization (2). The modulation of the gut microbiota by dietary means is being explored as a means of preserving and rescuing health and attention is turning to the potential role for probiotic organisms that are defined as "live microorganisms that, when administered in adequate amounts, confer a health benefit on the host" (3).

Interest in the anti-inflammatory properties of probiotics is growing particularly due to the potential benefits within the context of chronic inflammatory diseases (CID) where beneficial 
effects have been observed during rheumatoid arthritis, inflammatory bowel disease and obesity (to name but a few) $(4,5)$. A CID of particular concern is cardiovascular disease (CVD) that is a progressive inflammatory disease of the vasculature driven by hypercholesterolemia and is responsible for more than $30 \%$ of all global mortalities (6). A recent metaanalysis in high-risk subjects (with hypertension, excess body weight or hypercholesterolemia) concluded that probiotics were particularly effective for the reduction of low density lipoproteincholesterol (LDL-C) (7). It is now understood that elevated LDL-C can be a disease risk even in individuals with no history of CVD or any of its comorbidities (8) and that even marginal reductions in LDL-C profoundly lessen risk (9). Mechanistically, the anti-inflammatory capabilities of probiotics has been attributed to, at least in part, the generation of anti-inflammatory short chain fatty acids (SCFA) such as acetate and butyrate (5). The principle mechanism of cholesterol-lowering is related to inherent bile salt hydrolase (BSH) enzymes that deconjugate bile acids in the intestinal lumen rendering them less readily reabsorbed for recycling in the liver (10). The deconjugated bile acids are excreted from the body creating a bile acid deficit in the host that is counteracted by increased hepatic bile synthesis that utilises and therefore reduces circulatory cholesterol levels (11).

The Lab4 probiotic consortium comprises Bifidobacterium bifidum, Bifidobacterium animalis subsp. lactis and two strains of Lactobacillus acidophilus and anti-inflammatory capability has been observed in a previous study where blood mononuclear cells collected from Lab4 supplemented participants showed impaired secretion of interleukin (IL)- 6 and tumour necrosis factor (TNF)-alpha ex vivo (12). Assessment in silico of genomic composition identified genes encoding enzymes involved in the generation of SCFAs in all Lab4 strains (13). The cholesterollowering potential of Lab4 is better characterised and evidenced by (i) the ability to reduce both circulating LDL-C levels and bodyweight in hypercholesterolemic overweight and obese adults (14) and (ii) ameliorate the impact of high fat diet (HFD) induced elevations in plasma LDL-C and body weight in wildtype C57BL/6J mice (15). In atherosclerosis prone mice receiving a HFD, Lab4 contributed to the maintenance of plasma LDL-C levels and the inhibition of atherosclerotic plaque (fatty deposit) formation in the vasculature (16). All Lab4 organisms possess the gene sequence encoding the $\mathrm{BSH}$ enzyme choloylglycine hydrolase (13) and BSH activity has been observed during both assessments in vitro and in vivo (17). Studies in vitro show that the Lab4 consortium can assimilate cholesterol and regulate cholesterol transport across the intestinal epithelium (17).

This study details the impact of Lab4 on traditional CVD biomarkers (plasma cytokines/chemokines and lipids), plasma SCFAs and faecal bile acid profiles following a 90-day repeat feeding study in healthy wild-type Wistar rats.

\section{MATERIALS AND METHODS}

\section{Animal Maintenance and Probiotic Administration}

The study was performed within the test facility of INTOX Pvt. Ltd. (Maharashtra, India) in compliance with the OECD
Principles of Good Laboratory Practise [Organisation for Economic Co-operation and Development (OECD), 1998]. The animals in this study represent a subpopulation of a repeated dose 90-day oral toxicity study (13). Two groups of 10 male Wistar rats [6-7 weeks old, purchased from Vivo Bio Tech Ltd (Telangana, India)] were housed in pathogen-free ventilated cages (2 rats per cage) in a light- and temperature-controlled facility $(12 \mathrm{~h}$ light, $12 \mathrm{~h}$ dark, $19-25{ }^{\circ} \mathrm{C}$ ) and received a daily gavage of either the Lab4 probiotic comprising Lactobacillus acidophilus CUL21 (NCIMB 30156), Lactobacillus acidophilus CUL60 (NCIMB 30157), Bifidobacterium bifidum CUL20 (NCIMB 30153) and Bifidobacterium animalis subsp. lactis CUL34 (NCIMB 30172) at $3.0 \times 10^{10}$ colony forming units ( $\mathrm{cfu}$ ) in $10 \mathrm{~mL}$ phosphate buffered saline (PBS, Lab4 group) or a placebo comprising $10 \mathrm{~mL}$ sterile PBS (Control group) for 90 days. Throughout the study, rats were given access ad libitum to a standard diet [65 \% carbohydrate, $24 \%$ protein, $11 \%$ fat (Altromin 1320, Spezialfutter GmbH \& Co. KG, Germany)] and sterilised water in order to conform with animal welfare requirements. Body weight and food consumption were monitored weekly. At the end of the study, rats were sacrificed by exsanguination under $\mathrm{CO}_{2}$ asphyxiation.

\section{Blood Plasma Analysis}

Overnight fasted blood was collected into heparinised tubes at day 90. Plasma was extracted via centrifugation $(1,000$ $\mathrm{x} \mathrm{g}, 5 \mathrm{~min}$ ) and stored at $-80^{\circ} \mathrm{C}$ pending further analysis. Plasma concentrations of IL-4, IL-6, IL-10, IL-13, TNF- $\alpha$, interferon- $\gamma($ IFN- $\gamma)$ and keratinocyte chemoattractant/growth regulated oncogene $(\mathrm{KC} / \mathrm{GRO})$ were measured by the Central Biotechnology Service (CBS; Cardiff University, UK) using the VPLEX pro-inflammatory panel 1 rat kit (Meso Scale Discovery, Maryland, USA). Total cholesterol (TC), high-density lipoprotein cholesterol (HDL-C) and low-density lipoprotein cholesterol (LDL-C) and triglyceride (TG) concentrations were measured using Cholesterol and Triglyceride assay kits (ABCAM, Cambridge, UK) in accordance with the manufacturer's instructions. Total plasma bile acid levels were measured using the Xpand Plus Clinical Chemistry System (Siemens Healthcare Diagnostics Inc. Newark, U.S.A.) in accordance with the manufacturer's instructions. SCFA were measured using targeted gas chromatography and mass spectroscopy according to Moreau et al. (18).

\section{Faecal Bile Acid Analysis}

Faecal samples collected at day 90 were measured for bile acid content using ultra performance liquid chromatography-mass spectrometry (UPLC-MS), as previously described (19). Faecal pellets were lyophilized for $48 \mathrm{~h}$ (VirTis Benchtop BTP 8ZL freeze dryer, BPS FUK), weighed and then homogenised in a mixture of water, acetonitrile and 2-propanol (2:1:1 volumes) using a Biospec bead beater with $1.0 \mathrm{~mm}$ Zirconia beads. The homogenates were centrifuged at $16,000 \times \mathrm{g}$ for $20 \mathrm{~min}$ and the supernatant filtered through a $0.45 \mu \mathrm{m}$ membrane (Costar, Corning, UK). Bile acid analysis was performed on an ACQUITY UPLC (Waters Ltd, Elstree, UK) coupled to a Xevo G2 QToF mass spectrometer equipped with an electrospray ionisation 
source operating in negative ion mode and bile acid standards [55 bile acid standards including 36 non-conjugated, 12 conjugated with taurine, seven conjugated with glycine (Steraloids, Newport, RI)] were used to determine the chromatographic retention times. MassLynx software 4.1 was used for data acquisition. Relative faecal bile acid intensities were normalised to the faecal pellet dry weight. Principle component analysis (PCA) was carried out using SIMCA v14.1.0.2047 (MKS Umetrics, Umeå, Sweden) and heat-maps were generated in $\mathrm{R}$ package (Version 3.1.3).

\section{Analysis of Faecal Lactobacilli and Bifidobacteria}

Faecal samples were assessed for viable numbers of lactobacilli and bifidobacteria during the study of Baker et al. (13). Briefly, faeces were collected after 90 days intervention and 10-fold dilution series were set up in Maximum Recovery Diluent (MRD). Lactobacilli and bifidobacteria were plated on DeMan Rogosa Sharpe (MRS) medium or MRSX [MRS containing lithium chloride $(1 \mathrm{~g} / \mathrm{L})$, sodium propionate $(1.5 \mathrm{~g} / \mathrm{L})$ and Lcysteine hydrochloride $(0.25 \mathrm{~g} / \mathrm{L})$ ] respectively and incubated anaerobically (10\% carbon dioxide, $10 \%$ hydrogen and $80 \%$ nitrogen) at $37^{\circ} \mathrm{C}$. Bacterial species was confirmed by Gram staining, colony morphology and by analytical profile index (API, BioMerieux, Marcy-l'Étoile, France). Viable counts were recorded as the numbers of $\log _{10}$ colony forming units (cfus) per gram of sample.

\section{Statistical Analysis}

All data are presented as mean \pm standard deviation (SD) of the assigned number of rats. All data were assessed for homogeneity (Levene's test) and normality (Shapiro-Wilk test or inspection of Q-Q plots). For data that were non-normally distributed, log or Box-Cox transformations were performed. For single comparisons, values of $p$ were determined using an independent $t$-test (where data were normally distributed) or Wilcoxon-Mann test for non-parametric data. For multiple comparisons, values of $p$ were determined using one-way analysis of variance (ANOVA) with Dunnett's T3 post-hoc analysis or, where data were not normally distributed after log or BoxCox transformation, Kruskal-Wallis followed by Dunn's test with Bonferroni correction. All statistical tests were performed using GraphPad 7.0 (Prism). Where sufficient pairwise data was available, correlations were performed with a Spearman's rank coefficient analysis in R package (Version 3.1.3). Differences were considered significant when $p<0.05$.

\section{RESULTS}

\section{Plasma Inflammatory Markers}

Supplementation with the Lab4 probiotic resulted in a significant $37.05 \%$ reduction in plasma IL-6 $(p=0.026$, Table 1A), a significant $42.92 \%$ reduction in TNF- $\alpha(p=0.005)$ and a significant $38.53 \%$ reduction in $\mathrm{KC} / \mathrm{GRO}(p=0.036)$ compared to levels in the control. No changes were observed for the other inflammatory markers tested.
TABLE 1 | Plasma levels of inflammatory markers and lipids and faecal bile acid content in male Wistar rats after 90 days supplementation with Lab4.

\begin{tabular}{|c|c|c|}
\hline & Control & Lab4 \\
\hline \multicolumn{3}{|c|}{ A. Plasma inflammatory markers (pg/mL): } \\
\hline Interleukin-4 & $1.69 \pm 0.09$ & $1.67 \pm 0.16$ \\
\hline Interleukin-6 & $33.72 \pm 6.03$ & $21.23 \pm 9.75^{\star}$ \\
\hline Interleukin-10 & $9.27 \pm 1.45$ & $8.55 \pm 2.05$ \\
\hline Interleukin-13 & $5.71 \pm 1.38$ & $5.57 \pm 0.71$ \\
\hline Tumour necrosis factor- $\alpha$ & $4.66 \pm 1.76$ & $2.66 \pm 0.54^{\star *}$ \\
\hline Interferon- $\gamma$ & $1.90 \pm 0.40$ & $1.66 \pm 0.27$ \\
\hline $\begin{array}{l}\text { Keratinocyte chemoattractant/growth } \\
\text { regulated oncogene }\end{array}$ & $90.20 \pm 38.75$ & $55.46 \pm 18.25^{\star}$ \\
\hline \multicolumn{3}{|l|}{ B. Plasma lipids (mg/dL): } \\
\hline Total cholesterol (TC) & $70.20 \pm 12.15$ & $68.90 \pm 12.80$ \\
\hline $\begin{array}{l}\text { Low-density lipoprotein cholesterol } \\
\text { (LDL-C) }\end{array}$ & $42.98 \pm 8.04$ & $33.18 \pm 8.40^{*}$ \\
\hline $\begin{array}{l}\text { High-density lipoprotein cholesterol } \\
\text { (HDL-C) }\end{array}$ & $22.70 \pm 7.80$ & $32.85 \pm 10.67^{p=0.063}$ \\
\hline Triglycerides & $60.11 \pm 11.21$ & $72.78 \pm 18.03$ \\
\hline TC:HDL-C ratio & $3.71 \pm 1.66$ & $2.11 \pm 0.59^{*}$ \\
\hline LDL-C:HDL-C ratio & $2.26 \pm 1.29$ & $0.50 \pm 0.23^{\star \star}$ \\
\hline \multicolumn{3}{|c|}{ C. Faecal bile acid content (as a ratio of control group): } \\
\hline Total bile acids & NA & $1.14 \pm 0.18^{p=0.063}$ \\
\hline Conjugated bile acids & NA & $0.97 \pm 0.41$ \\
\hline Unconjugated bile acids & NA & $1.14 \pm 0.19^{p=0.052}$ \\
\hline Conjugate/Unconjugated ratio & NA & $0.86 \pm 0.38$ \\
\hline Cholic acid & NA & $1.60 \pm 0.40^{*}$ \\
\hline
\end{tabular}

Data represents the mean $\pm S D$ of 6 to 10 rats per group $(A)$ or 10 rats per group ( $B$ and C). Values of $p$ were ${ }^{*} p<0.05$ or $^{\star \star} p<0.05$ or as stated compared to the control group. NA, not applicable.

\section{Plasma SCFA}

Plasma SCFA concentrations were comparable between groups (Figure 1) although levels in the Lab4 group were consistently higher than in the control group: valeric acid $(0.56 \pm 0.21 v s$. $0.38 \pm 0.34 \mathrm{pg} / \mathrm{ml}$ respectively, $47.4 \%$ increase), acetic acid ( $14.04 \pm 5.64$ vs. $9.56 \pm 4.74 \mathrm{pg} / \mathrm{ml}$ respectively, $46.9 \%$ increase), isobutyric acid (1.46 \pm 0.51 vs. $1.16 \pm 0.46 \mathrm{pg} / \mathrm{ml}$ respectively, $25.9 \%$ increase), butyric acid ( $1.65 \pm 0.52$ vs. $1.36 \pm 0.50 \mathrm{pg} / \mathrm{ml}$ respectively, $21.3 \%$ increase), isovaleric acid ( $0.16 \pm 0.04 v s$. $0.15 \pm 0.59 \mathrm{pg} / \mathrm{ml}$ respectively, $6.7 \%$ increase) and propionic acid $(18.59 \pm 4.57$ vs. $18.31 \pm 7.68 \mathrm{pg} / \mathrm{ml}$ respectively, 1.5 $\%$ increase).

\section{Plasma Lipids}

Plasma total cholesterol (TC) and triglycerides (TG) were similar between groups after 90 days feeding. LDL-C was significantly lower $(-22.8 \%, p=0.029$, Table 1B) and HDL$\mathrm{C}$ was higher $(44.7 \%, p=0.063)$ in the Lab4 group compared to the control. Between group ratios of LDL-C:HDLC $(-50.4 \%, p=0.007)$ and TC:HDL-C $(-36.8 \%, p=$ 0.028 ) were significantly lower for the Lab4 group. Bodyweights and food consumption rates did not differ between groups (Supplementary Figure S1). 

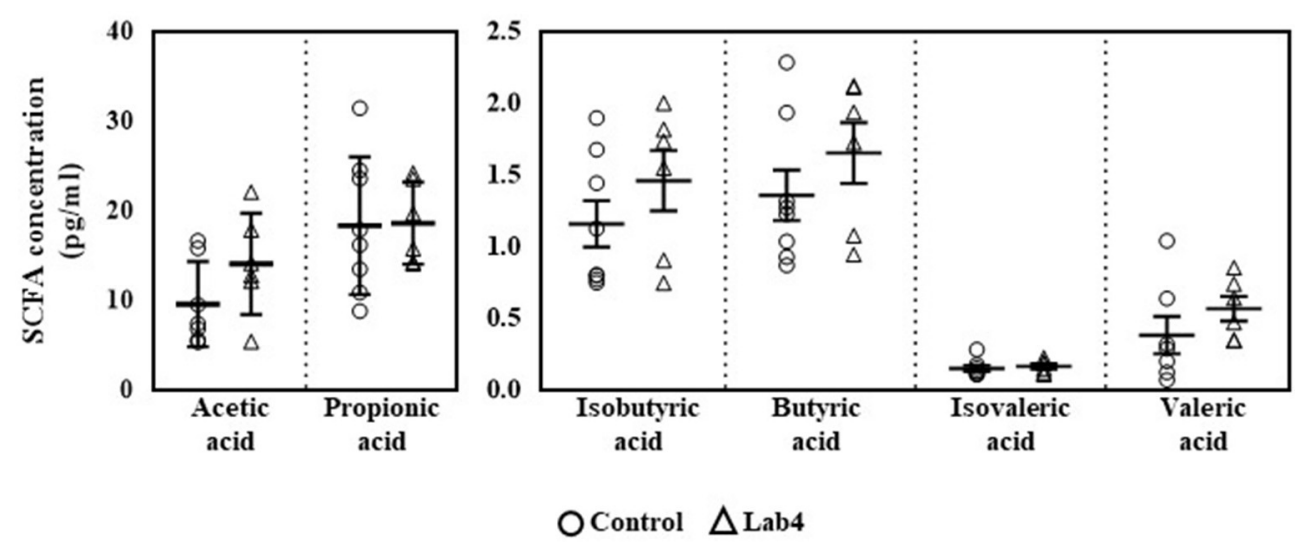

FIGURE 1 | Plasma levels of SCFA in Wistar rats after 90 days supplementation. Plasma levels of SCFA were quantified in control or Lab4 supplemented rats. Data represent the means $\pm S D$ of six to eight rats per group. SCFA, short chain fatty acid.

A

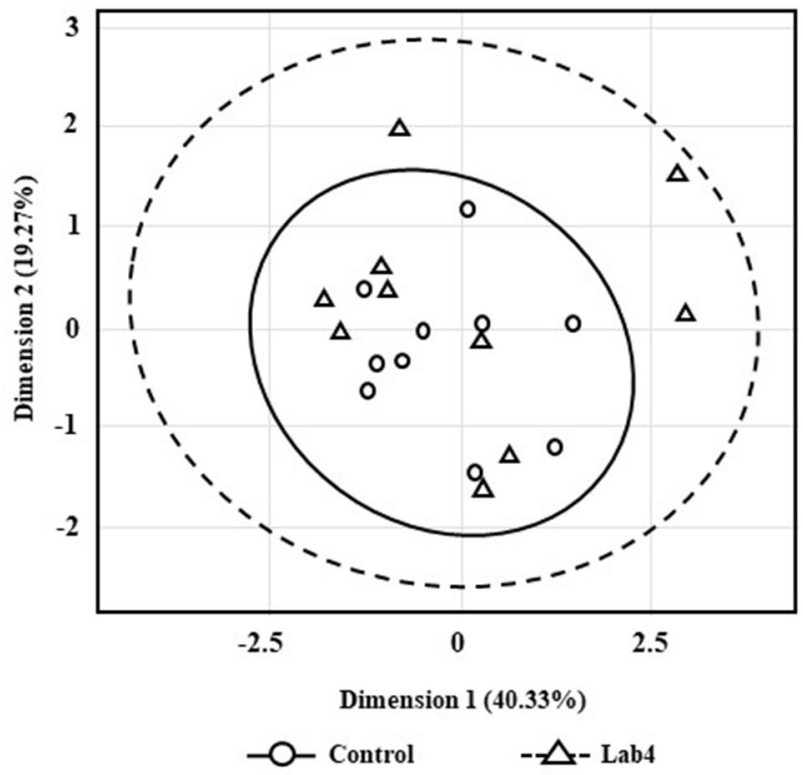

B
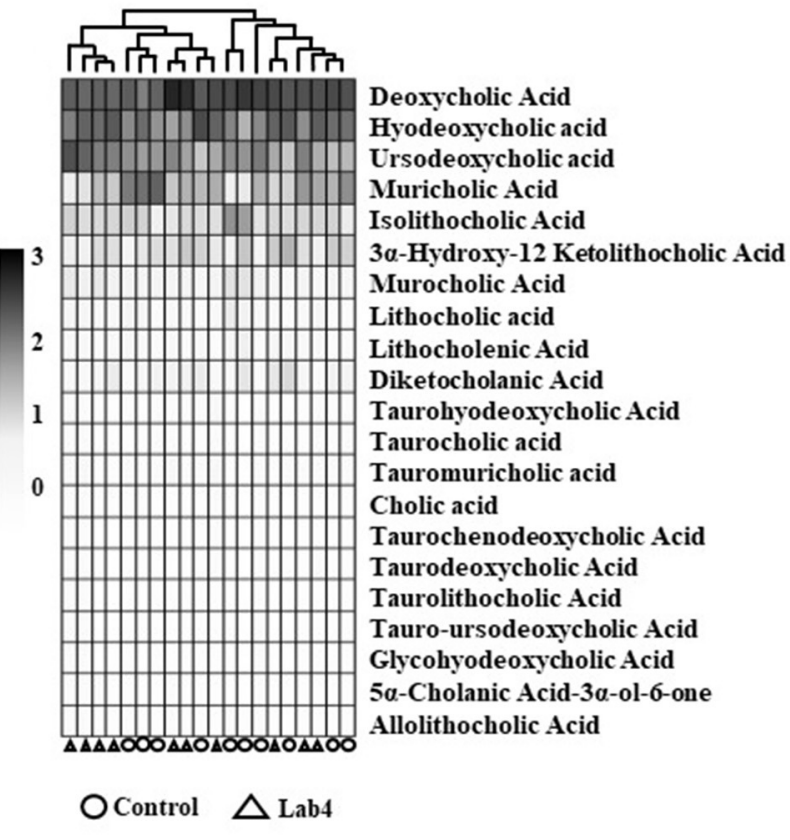

FIGURE 2 | Faecal bile acid profiles of Wistar rats after 90 days supplementation. (A) Principle component analysis (PCA) scores plot of bile acid signatures from control or Lab4 supplemented rats or $\mathbf{B}$ ) heat-map of the bile acid relative intensity for each rat. Analysis was performed on 10 rats per group.

\section{Faecal Bile Acid Profiling}

No significant differences were observed between groups in the faecal bile acid profiles whether by Principle Component Analysis (PCA; Figure 2A) or heat-map analysis (Figure 2B) but a more variable spatial organisation was observed for the Lab4 group (Figure 2A). Univariate analysis detected $14 \%$ higher levels of both total and unconjugated bile acids $(p=0.063$ and $p=0.052$, respectively, Table 1C) and $60 \%$ higher levels of cholic acid $(p=0.043)$ in the Lab4 group compared to the control. No further significant differences were observed (Supplementary Figure S2) and total bile concentrations in blood plasma were consistent between groups (data not shown).

\section{Spearman's Ranked Correlation Analysis}

Correlative analysis was performed for plasma cytokines/chemokines, plasma SCFAs, plasma lipids, faecal bile acids and faecal numbers of viable lactobacilli and bifidobacteria [measured by Baker et al. (13), data shown in Supplementary Figure S3]. TNF- $\alpha$ levels correlated negatively with faecal levels of Bifidobacterium $(R=-0.94, p=0.0048$, 


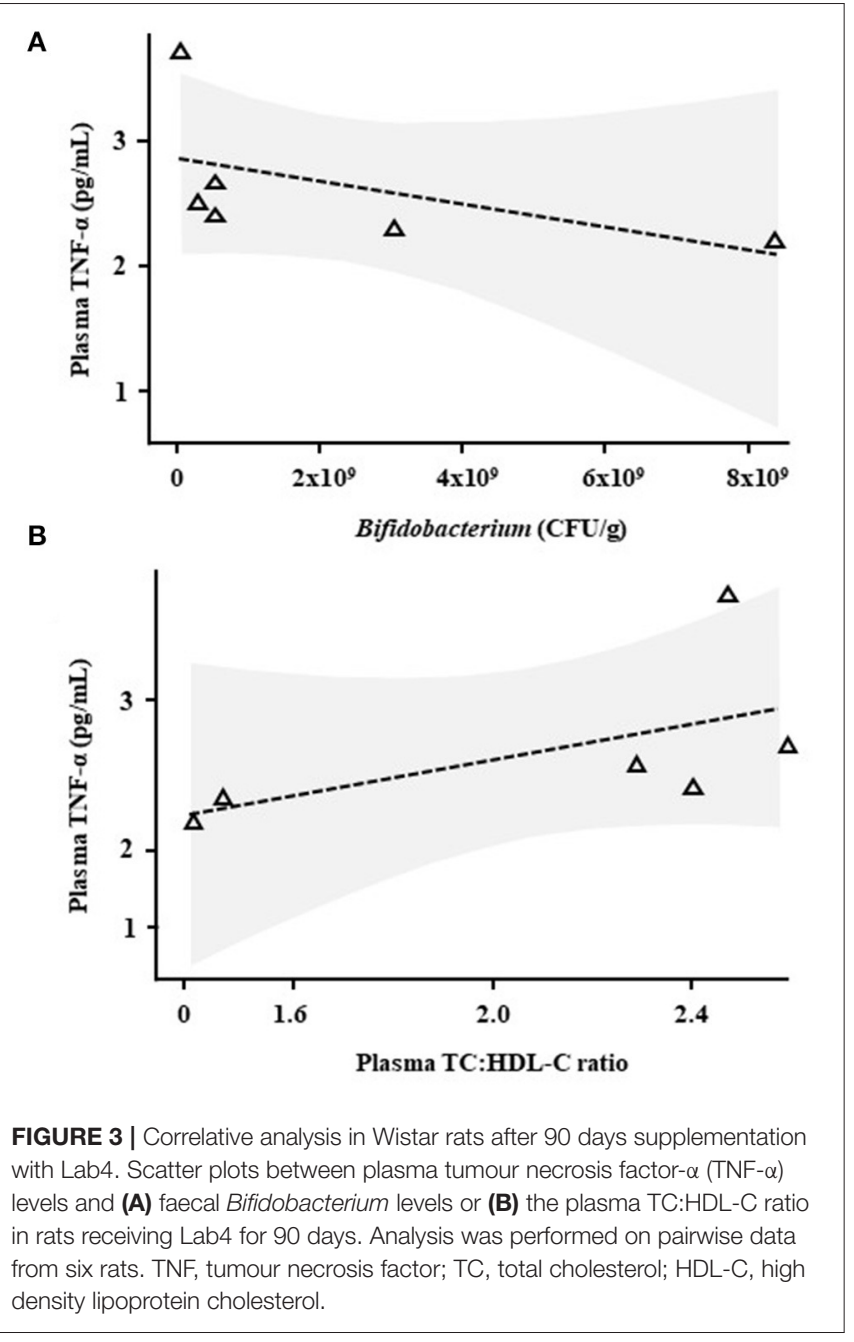

Figure 3A) and positively with plasma TC:HDL-C $(R=0.89, p$ $=0.019$, Figure 3B) only in the Lab4 group.

\section{DISCUSSION}

During this 90-day feeding study in wild-type Wistar rats, daily supplementation with the Lab4 probiotic imparted an antiinflammatory effect by reducing plasma levels of IL-6, TNF- $\alpha$ and $\mathrm{KC} / \mathrm{GRO}$ and improved plasma cholesterol profiles, possibly via the enhancement of bile acid deconjugation in the intestines.

Elevated levels of pro-inflammatory cytokines have been detected in healthy asymptomatic individuals and are thought to be triggered by many factors including stress, poor diet, physical inactivity and lack of sleep (20). Plasma analysis of the Lab4 supplemented rats revealed significant reductions in the pro-inflammatory cytokines IL-6 and TNF- $\alpha$ and the pro-inflammatory chemokine KC/GRO implying an antiinflammatory capability. These findings align with the outcomes from a previous study where analysis ex vivo of blood mononuclear cells collected from Lab4 supplemented healthy adult participants showed reduced secretion of IL- 6 and TNF- $\alpha$
(12). Other probiotic studies have demonstrated the ability of bifidobacteria and/or lactobacilli to reduce plasma levels of IL-6 and/or TNF- $\alpha$ in pre-clinical animal models (2123) and human subjects (24) and Lactobacillus fermentum JDFM216 supplementation reduced serum KC/GRO in ageing mice (25). Inflammation is a major risk factor of CVD and a myriad of pro-inflammatory cytokines and chemokines have been associated with this disease progression (26). IL-6 and TNF- $\alpha$ are predominantly produced by cells of the innate immune system including monocytes, macrophages and natural killer cells in response to a physiological challenge (e.g., oxidative stress) (27). Pharmacological inhibition of their activity is considered a promising approach to reduce CVD risk $(28,29)$.

The anti-inflammatory capabilities of probiotics have been linked, at least in part, with the generation of SCFAs such as acetic, propionic and butyric acid, via the fermentation of dietary fibre (30). In rats receiving Lab4, a general pattern of increase was observed for each SCFA measured with notable changes occurring for valeric, acetic and butyric acids. Each strain of the Lab4 consortium possesses gene sequences encoding phosphate acetyltransferase and acetate kinase that are involved in the generation of acetate (31): acetate is recognised as an anti-inflammatory SCFA known to inhibit the expression of IL- 6 by colon cells (32). The recent study of Yang et al. showed that administration of Lactobacillus fermentum ZJUIDS06 increased levels of acetic, propionic and butyric acid in the colons of hypercholesterolemic hamsters whilst significantly reducing plasma cholesterol levels (33). SCFA are known to have pleiotropic effects on the host and there is growing evidence supporting beneficial effects on cholesterol metabolism (34).

Hypercholesterolemia is a major risk factor for CVD (35) and reductions in circulating cholesterol levels, particularly LDL-C, are encouraged to reduce the risk of CVD (36) even in overtly healthy subjects (8). In this study with Lab4 supplemented rats we showed a $22.8 \%$ reduction in the plasma LDL-C concentration even in the absence of any hypercholesteraemic profile. Such outcomes could imply a potential cardiovascular benefit and expand on the outcomes of our own studies with Lab4 $(13,14$, $16,17)$ whilst adding to the growing evidence supporting an LDL-C lowering capability by probiotics (7). In contrast, due to their function in the disposal of excess cholesterol via the liver, high circulating HDL-C levels are linked to a reduced CVD risk (37). In the probiotic fed rats, plasma HDL-C levels were $44 \%$ higher than in the control group - an outcome consistent with findings observed in a study with Lab4 supplemented atherosclerosis prone mice (16). In both studies, the changes in lipid profiles contributed to significant reductions in the TC:HDL and LDL:HDL ratios in the animals receiving Lab4 compared to the controls. The TC:HDL and LDL:HDL ratios are clinically recognised markers of cardiovascular risk (37).

Reduction of cholesterol by probiotic bacteria can involve the deconjugation of bile acids in the intestinal lumen which is mediated by the actions of inherent BSH enzymes (11). The Lab4 consortium has BSH activity $(13,17)$ and increased excretion rates of deconjugated bile acids (both 
total and cholic) were seen with Lab4 treated Wistar rats indicating an ability to impact upon bile acid metabolism during normal metabolic processes and consistent with the reductions observed in circulating LDL-C. The ability of Lab4 to assimilate cholesterol and regulate the transport of cholesterol across the intestinal epithelium (17) may have contributed to its hypocholesterolaemic effects and represents a avenue of future research.

Correlative analyses showed that plasma levels of TNF- $\alpha$ were negatively associated with numbers of faecal bifidobacteria in the Lab4 supplemented rats. As previously mentioned, anti-inflammatory activity is recognised in bifidobacteria (38), the Lab4 consortium comprises Bifidobacterium bifidum and Bifidobacterium animalis subsp. lactis and increased levels of viable bifidobacteria (and lactobacilli) were observed in the faeces of the rats receiving Lab4 (Supplementary Figure S3). In addition, TNF- $\alpha$ levels positively correlated with the plasma TC:HDL-C ratio potentially linking circulating TNF- $\alpha$ and CVD development (39).

There are a number of limitations of the study. Only healthy male rats receiving a single dose of probiotic were included in the analysis. Future studies should assess the cardiovascular and inflammatory impact of different doses of Lab4 in healthy male and female subjects and in cohorts with high risk of cardiovascular disease.

In summary, 90-day supplementation of rats with the Lab4 probiotic resulted in significant reductions in circulating levels of IL-6, TNF- $\alpha$ and KC/GRO and improvements in circulating lipid profiles, possibly mediated by the increased excretion of bile acids. This study highlights daily supplementation with the Lab4 consortium as a potential prophylactic against CVD and has provided support for future human intervention studies.

\section{DATA AVAILABILITY STATEMENT}

The raw data supporting the conclusions of this article will be made available by the authors, without undue reservation.

\section{REFERENCES}

1. Fan Y, Pedersen O. Gut microbiota in human metabolic health and disease. Nat Rev Microbiol. (2021) 19:55-71. doi: 10.1038/s41579-020-0433-9

2. Vijay A, Astbury S, Panayiotis L, Marques FZ, Spector TD, Menni C, et al. Dietary interventions reduce traditional and novel cardiovascular risk markers by altering the gut microbiome and their metabolites. Front Cardiovasc Med. (2021) 8:691564. doi: 10.3389/fcvm.2021.691564

3. FAO/WHO. Probiotics in food: health and nutritional properties and guidelines for evaluation: report of a Joint FAO/WHO Expert Consultation on Evaluation of Health and Nutritional Properties of Probiotics in Food, including powder milk with live lactic acid bacteria: Cordoba, Argentina, 14 October 2001: report of a Joint FAO/WHO Working Group on Drafting Guidelines for the Evaluation of Probiotics in Food. Rome: Food and Agriculture Organization of the United Nations. (2006).

4. Liu Y, Alookaran JJ, Rhoads JM. Probiotics in autoimmune and inflammatory disorders. Nutrients. (2018) 10:1537. doi: 10.3390/nu10 101537

5. Cristofori F, Dargenio VN, Dargenio C, Miniello VL, Barone M, Francavilla R. Anti-inflammatory and immunomodulatory effects of probiotics in gut

\section{ETHICS STATEMENT}

The animal study was reviewed and approved by the Institutional Ethics Committee of INTOX Pvt. Ltd. (Maharashtra, India) who are a specialist animal testing facility compliant with all local ethical regulations and with the OECD Principles of Good Laboratory Practice [Organisation for Economic Co-operation and Development (OECD), 1998].

\section{AUTHOR CONTRIBUTIONS}

SFP, TSW, and DRM were responsible for the design of the study. Experiments were performed by TSW, LMB, and AAJ. Data and statistical analyses were performed by GM, DRM, and TSW. TRH, JRM, GR, and PDF provided assistance and knowledge that was vital to the completion of the manuscript. DRM, JD, and SFP prepared the manuscript. All authors contributed to the review of the manuscript.

\section{FUNDING}

JRM and the Department of Metabolism, Digestion, and Reproduction at Imperial College London received funding from the National Institute of Health Research (NIHR), the Biomedical Research Centre (BRC) based at Imperial College London, and Imperial College Healthcare NHS Trust.

\section{ACKNOWLEDGMENTS}

The authors would like to acknowledge scientists at INTOX Pvt. Ltd. for assistance during the study and Dr. Alex Pechlivanis of Imperial College London for his technical assistance.

\section{SUPPLEMENTARY MATERIAL}

The Supplementary Material for this article can be found online at: https://www.frontiersin.org/articles/10.3389/fnut.2021. 778289/full\#supplementary-material inflammation: a door to the body. Front Immunol. (2021) 12:578386. doi: 10.3389/fimmu.2021.578386

6. Olas B. Probiotics, prebiotics and synbiotics-a promising strategy in prevention and treatment of cardiovascular diseases? Int J Mol Sci. (2020) 21:9737. doi: 10.3390/ijms21249737

7. Dixon A, Robertson K, Yung A, Que M, Randall H, Wellalagodage D, et al. Efficacy of probiotics in patients of cardiovascular disease risk: a systematic review and meta-analysis. Curr Hypertens Rep. (2020) 22:74. doi: 10.1007/s11906-020-01080-y

8. Abdullah SM, Defina LF, Leonard D, Barlow CE, Radford NB, Willis $\mathrm{BL}$, et al. Long-term association of low-density lipoprotein cholesterol with cardiovascular mortality in individuals at low 10-year risk of atherosclerotic cardiovascular disease. Circulation. (2018) 138:2315-25. doi: 10.1161/CIRCULATIONAHA.118.034273

9. Silverman MG, Ference BA, Im K, Wiviott SD, Giugliano RP, Grundy SM, et al. Association between lowering ldl-c and cardiovascular risk reduction among different therapeutic interventions: a systematic review and meta-analysis. JAMA. (2016) 316:1289-97. doi: 10.1001/jama.2016.13985

10. Kumar M, Nagpal R, Kumar R, Hemalatha R, Verma V, Kumar A, et al. Cholesterol-lowering probiotics as potential biotherapeutics for 
metabolic diseases. Exp Diabetes Res. (2012) 2012:902917. doi: 10.1155/2012/9 02917

11. Sivamaruthi BS, Fern LA, Rashidah Pg Hj Ismail DSN, Chaiyasut C. The influence of probiotics on bile acids in diseases and aging. Biomed Pharmacother. (2020) 128:110310. doi: 10.1016/j.biopha.2020.110310

12. Hepburn NJ, Garaiova I, Williams EA, Michael DR, Plummer S. Probiotic supplement consumption alters cytokine production from peripheral blood mononuclear cells: a preliminary study using healthy individuals. Benef Microbes. (2013) 4:313-7. doi: 10.3920/BM2013.0012

13. Baker LM, Davies TS, Masetti G, Hughes TR, Marchesi JR, Jack AA, et al. A genome guided evaluation of the Lab4 probiotic consortium. Genomics. (2021). doi: 10.1016/j.ygeno.2021.08.007

14. Michael DR, Jack AA, Masetti G, Davies TS, Loxley KE, Kerry-Smith J, et al. A randomised controlled study shows supplementation of overweight and obese adults with lactobacilli and bifidobacteria reduces bodyweight and improves well-being. Sci Rep. (2020) 10:4183. doi: 10.1038/s41598-020-60991-7

15. Thaiss CA, Itav S, Rothschild D, Meijer MT, Levy M, Moresi C, et al. Persistent microbiome alterations modulate the rate of post-dieting weight regain. Nature. (2016) 540:544. doi: 10.1038/nature20796

16. O’Morain VL, Chan Y-H, Williams JO, Alotibi R, Alahmadi A, Rodrigues $\mathrm{NP}$, et al. The Lab4P consortium of probiotics attenuates atherosclerosis in LDL receptor deficient mice fed a high fat diet and causes plaque stabilization by inhibiting inflammation and several pro-atherogenic processes. Mol Nutr Food Res. (2021) 65:2100214. doi: 10.1002/mnfr.202100214

17. Michael DR, Davies TS, Moss JWE, Calvente DL, Ramji DP, Marchesi JR, et al. The anti-cholesterolaemic effect of a consortium of probiotics: An acute study in C57BL/6J mice. Sci Rep. (2017) 7:2883. doi: 10.1038/s41598-017-02889-5

18. Moreau NM, Goupry SM, Antignac JP, Monteau FJ, Le Bizec BJ, Champ $\mathrm{MM}$, et al. Simultaneous measurement of plasma concentrations and 13C-enrichment of short-chain fatty acids, lactic acid and ketone bodies by gas chromatography coupled to mass spectrometry. $J$ Chromatogr B Analyt Technol Biomed Life Sci. (2003) 784:395-403. doi: 10.1016/S1570-0232(02)00827-9

19. Sarafian MH, Lewis MR, Pechlivanis A, Ralphs S, McPhail MJ, Patel VC, et al. Bile acid profiling and quantification in biofluids using ultra-performance liquid chromatography tandem mass spectrometry. Anal Chem. (2015) 87:9662-70. doi: 10.1021/acs.analchem.5b01556

20. Hostinar CE, Ross KM, Chen E, Miller GE. Modeling the association between lifecourse socioeconomic disadvantage and systemic inflammation in healthy adults: The role of self-control. Health Psychol. (2015) 34:580-90. doi: $10.1037 /$ hea0000130

21. Salazar N, Lopez P, Garrido P, Moran J, Cabello E, Gueimonde M, et al. Immune modulating capability of two exopolysaccharide-producing Bifidobacterium strains in a Wistar rat model. Biomed Res Int. (2014) 2014:106290. doi: 10.1155/2014/106290

22. Zhang X, Kang Y, Xie Z, Su J, Kong X, editors. Oral administration of Lactobacillus acidophilus stain SW 1 suppresses tumor necrosis factor (TNF)alpha and increases transforming growth factor (TGF)-beta in mice2016.

23. Karamese M, Aydin H, Sengul E, Gelen V, Sevim C, Ustek D, et al. The Immunostimulatory Effect of Lactic Acid Bacteria in a Rat Model. Iran J Immunol. (2016) 13:220-8.

24. Milajerdi A, Mousavi SM, Sadeghi A, Salari-Moghaddam A, Parohan M, Larijani B, et al. The effect of probiotics on inflammatory biomarkers: A meta-analysis of randomized clinical trials. Eur J Nutr. (2020) 59:633-49. doi: 10.1007/s00394-019-01931-8

25. Park MR, Shin M, Mun D, Jeong SY, Jeong DY, Song M, et al. Probiotic Lactobacillus fermentum strain JDFM216 improves cognitive behavior and modulates immune response with gut microbiota. Sci Rep. (2020) 10:21701. doi: 10.1038/s41598-020-77587-w

26. Williams JW, Huang LH, Randolph GJ. Cytokine circuits in cardiovascular disease. Immunity. (2019) 50:941-54. doi: 10.1016/j.immuni.2019.03.007

27. Lacy P, Stow JL. Cytokine release from innate immune cells: association with diverse membrane trafficking pathways. Blood. (2011) 118:9-18. doi: 10.1182/blood-2010-08-265892
28. Ma J, Chen X. Anti-inflammatory therapy for coronary atherosclerotic heart disease: unanswered questions behind existing successes. Front Cardiovasc Med. (2020) 7:631398. doi: 10.3389/fcvm.2020.631398

29. Michael S, Tanveer T. The effect of TNF $\alpha$-inhibitors on cardiovascular events in patients with rheumatoid arthritis: an updated systematic review of the literature. Curr Rheumatol Rev. (2016) 12:208-22. doi: 10.2174/1573397112666160404124655

30. Markowiak-Kopec P, Slizewska K. The effect of probiotics on the production of short-chain fatty acids by human intestinal microbiome. Nutrients. (2020) 12:1107. doi: 10.3390/nu12041107

31. Eiteman MA, Altman E. Overcoming acetate in Escherichia coli recombinant protein fermentations. Trends Biotechnol. (2006) 24:530-6. doi: 10.1016/j.tibtech.2006.09.001

32. Tedelind S, Westberg F, Kjerrulf M, Vidal A. Anti-inflammatory properties of the short-chain fatty acids acetate and propionate: a study with relevance to inflammatory bowel disease. World J Gastroenterol. (2007) 13:2826-32. doi: 10.3748/wjg.v13.i20.2826

33. Yang D, Lyu W, Hu Z, Gao J, Zheng Z, Wang W, et al. Probiotic Effects of Lactobacillus fermentum ZJUIDS06 and Lactobacillus plantarum ZY08 on Hypercholesteremic Golden Hamsters. Front Nutr. (2021) 8:705763. doi: 10.3389/fnut.2021.705763

34. Villette R, Kc P, Beliard S, Salas Tapia MF, Rainteau D, Guerin M, et al. Unraveling host-gut microbiota dialogue and its impact on cholesterol levels. Front Pharmacol. (2020) 11:278. doi: 10.3389/fphar.2020.00278

35. McLaren JE, Michael DR, Ashlin TG. Ramji DPJPilr. Cytokines, macrophage lipid metabolism and foam cells: implications for cardiovascular disease therapy. Prog Lipid Res. (2011) 50:331-47. doi: 10.1016/j.plipres.2011. 04.002

36. Arnett DK, Blumenthal RS, Albert MA, Buroker AB, Goldberger ZD, Hahn EJ, et al. 2019 ACC/AHA guideline on the primary prevention of cardiovascular disease: a report of the American College of Cardiology/American Heart Association task force on clinical practice guidelines. Circulation. (2019) 140:e596-646. doi: 10.1161/CIR.0000000000000678

37. Millan J, Pinto X, Munoz A, Zuniga M, Rubies-Prat J, Pallardo LF, et al. Lipoprotein ratios: Physiological significance and clinical usefulness in cardiovascular prevention. Vasc Health Risk Manag. (2009) 5:757-65. doi: 10.2147/VHRM.S6269

38. Ruiz L, Delgado S, Ruas-Madiedo P, Sanchez B, Margolles A. Bifidobacteria and their molecular communication with the immune system. Front Microbiol. (2017) 8:2345. doi: 10.3389/fmicb.2017.02345

39. Bradley JR. TNF-mediated inflammatory disease. J Pathol. (2008) 214:149-60. doi: $10.1002 /$ path.2287

Conflict of Interest: TSW, GM, JD, AAJ, SFP, and DRM were employed by the company Cultech Limited.

The remaining authors declare that the research was conducted in the absence of any commercial or financial relationships that could be construed as a potential conflict of interest.

Publisher's Note: All claims expressed in this article are solely those of the authors and do not necessarily represent those of their affiliated organizations, or those of the publisher, the editors and the reviewers. Any product that may be evaluated in this article, or claim that may be made by its manufacturer, is not guaranteed or endorsed by the publisher.

Copyright (C) 2021 Webberley, Masetti, Baker, Dally, Hughes, Marchesi, Jack, Plummer, Ramanathan, Facey and Michael. This is an open-access article distributed under the terms of the Creative Commons Attribution License (CC BY). The use, distribution or reproduction in other forums is permitted, provided the original author(s) and the copyright owner(s) are credited and that the original publication in this journal is cited, in accordance with accepted academic practice. No use, distribution or reproduction is permitted which does not comply with these terms. 